\title{
Rigenerazione e proteinuria, quando l'albumina fa la differenza
}

\author{
Duccio Lombardi
}

Dottorando in Area del Farmaco e Trattamenti Innovativi, Dipartimento di Medicina Sperimentale e Clinica, Centro DENOTHE, Università degli Studi di Firenze, Firenze

\begin{abstract}
REgENERATION AND PROTEINURIA, WHEN ALBUMin MAKES THE DIFFERENCE
Abstract. In chronic kidney disease (CKD) the risk of organ failure is correlated with the severity of proteinuria, which, in its turns, depends on the number of lost podocytes and on the consequent glomerular fibrosis. Nevertheless, several experimental and clinical evidences suggest the possibility of remission of kidney diseases, and in some cases even of regression of the damage, at least if the histology of the organ is not totally compromised.

Particularly, these results can be achieved by employing antiproteinuric pharmacological modulators.

New experimental evidences suggest why blocking the leakage of proteins in urine is able to allow the remission of CKD. In a recent paper, Peired et al documented for the first time how albuminuria can halt the regenerative process via the sequestration of retinoic acid (RA) by albumin itself. RA is, in fact, a well-know differentiative agent for stem cells' and progenitor cells' populations located in various organs.

The consequent urinary loss of RA bound to albumin prevents the activation of renal progenitors resident along the Bowman's capsule, blocking in its first phases the regenerative response, and thus allowing the progression of the CKD.
\end{abstract}

Key words: Kidney Regeneration, Podocyte regeneration, Albuminuria, Retinoic Acid

Conflict of interest: None.

Financial support: None.

Accettato: 17 Febbraio 2014

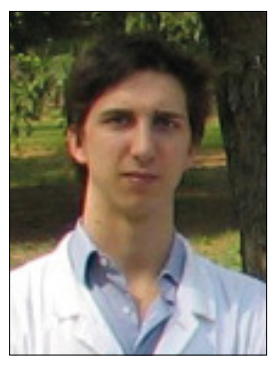

Duccio Lombardi

\section{Introduzione}

Da molto tempo, la proteinuria, che si presenta soprattutto sotto forma di albuminuria, è nota per la sua correlazione a danni renali, ma soltanto da poco è stata accettata dalla comunità scientifica come segno prognostico nell'evoluzione della malattia renale cronica $(\mathrm{CKD}$, Chronic Kidney Disease).

A partire dagli anni '80, numerosi studi hanno evidenziato come la proteinuria sia causa di alterazioni morfologiche podocitarie come perdita dei processi pedicellari, aumento del volume del corpo cellulare, formazione di inclusioni proteiche citoplasmatiche e vacuolizzazione (1-3). Queste modifiche cui il podocita va incontro furono immediatamente associate a una disfunzione dello slit diaphragm, evento che causa distacco e morte del podocita stesso in concomitanza con la regressione dei suoi processi pedicellari. Tutti questi eventi sono direttamente correlati alla perdita irreversibile della normale permeabilità della barriera di filtrazione glomerulare (4) e indirizzano il paziente verso la malattia renale terminale (ESRD, End Stage Renal Disease).
Quanto emerso più recentemente è che la proteinuria, alterazione riscontrabile nella quasi totalità delle nefropatie, non è un semplice e importante indice patologico e prognostico, ma risulta anche essere il principale fattore che contribuisce all'evoluzione della nefropatia. La proteinuria instaura, infatti, un circolo vizioso che progressivamente aggrava l'insulto iniziale $(5,6)$. Il danno del podocita, indotto dalla proteinuria e in particolar modo dall'albuminuria, innesca infatti un "effetto domino" che concorre sia alla progressiva deplezione dei podociti inizialmente rimasti indenni a seguito dell'insulto originario, sia al graduale incremento della proteinuria. La perdita di proteine causa danno e, conseguentemente, morte del podocita, evento che a sua volta conduce a un'ulteriore fuga di proteine.

Numerosi studi, al contempo, evidenziano come il danno podocitario sia reversibile. Recentemente è stata individuata la presenza di una popolazione di progenitori, residenti presso il polo urinario della capsula di Bowman (RPC, Renal Progenitor Cells), capaci di differenziare a podocita $(7,8)$ e di sostituire, in seguito a un insulto, tali cellule terminalmente differenziate e incapaci di divisione.

Analogamente, è stato dimostrato come la remissione della malattia renale sia possibile e, in particolare, otte- 
nibile mediante terapie farmacologiche con effetti antiproteinurici $(9,10)$. Quindi, se i podociti possono essere sostituiti, perché questo non avviene automaticamente in seguito a un danno che determina la perdita di tali cellule? Perché solo nel caso di somministrazione di terapie antiproteinuriche si può ottenere la regressione delle lesioni glomerulari?

Queste due domande lasciano ipotizzare che la proteinuria possieda un effetto dannoso diretto sulle RPC o che indirettamente riesca a bloccare la rigenerazione glomerulare, impedendo ai progenitori renali di attivarsi.

\section{Nuove evidenze sull'albuminuria}

Peired et al. hanno recentemente dato risposta a questi interrogativi in un articolo (11) pubblicato sulla rivista Journal of the American Society of Nephrology. Nello studio, gli Autori hanno esposto colture di RPC alle principali proteine che sono perse in caso di alterazione della barriera di filtrazione glomerulare, quali transferrina, IgG e albumina. In tal modo è stato possibile dimostrare che, mentre $\operatorname{IgG}$ e transferrina non alterano le capacità dei progenitori renali di differenziare verso il lineage podocitario, al contrario la presenza della sola albumina è in grado di impedire alle RPC di acquisire marcatori tipici del podocita come nefrina, podocina, podocalixina e p21.

Successivamente, nel paper (11) sono stati analizzati i singoli componenti che costituiscono il terreno di coltura VRAD, impiegato per indurre il differenziamento delle RPC a podociti, al fine di capire quali siano i costituenti che inducono l'acquisizione del fenotipo podocitario. Da tale analisi, è emerso che l'effetto differenziativo è prevalentemente dovuto alla presenza di acido retinoico (metabolita attivo della vitamina A), ma che, in presenza di albumina, gli effetti dell'acido retinoico (RA, Retinoic Acid) sono aboliti, impedendo, così, alle RPC di divenire podociti.

Questo effetto è dovuto alla capacità dell'albumina di legare e sequestrare l'acido retinoico, negando il suo ingresso nella cellula, evento necessario ad attivare il programma genico che porta al differenziamento in senso podocitario. Il fenomeno è stato in particolare dimostrato impiegando VRAD contenente RA radio-marcato in presenza di concentrazioni sempre maggiori di albumina. Da tale analisi è emerso che, all'aumentare della concentrazione di albumina, sempre meno acido retinoico è in grado di garantirsi l'accesso all'ambiente intracellulare, rimanendo nel terreno di coltura. Al contempo, però, le RPC sono in grado di sintetizzare endogenamente acido retinoico partendo dal retinolo grazie all'enzima retinaldeide deidrogenasi. Se, quindi, il RA non può garantirsi, in condizioni patologiche, l'accesso alle RPC, tali cellule dovrebbero comunque essere in grado di sintetizzarlo partendo dal retinolo comunemente presente a livello plasmatico. A conferma di ciò, gli Autori hanno dimostrato che il trattamento dei progenitori renali con retinolo induce il differenzieranno in senso podocitario; ma se, assieme al retinolo, viene aggiunta in coltura anche l'albumina, la capacità di acquisire marker tipici podocitari viene meno. Ciò suggerisce che l'albumina possa entrare all'interno dei progenitori renali sequestrando anche l'acido retinoico sintetizzato endogenamente.

È, perciò, possibile affermare che l'albumina è capace di bloccare il differenziamento delle RPC a podocita mediante sequestro dell'acido retinoico, molecola necessaria affinché siano trascritti specifici geni podocitari, come dimostrato nello stesso studio (11).

Successivamente, Peired et al. (11) hanno valutato l'effetto dell'albuminuria e della somministrazione di acido retinoico sulla rigenerazione podocitaria in vivo. Impiegando come modello la nefropatia da adriamicina (Adriamycin Nephropathy, AN), è stato ricreato l'analogo sperimentale della glomerulosclerosi focale segmentaria umana (Focal Segmental GlomeruloSclerosis, FSGS) in topi SCID (Severe Combined ImmunoDeficency). La scelta di tale modello murino trova il suo razionale in quanto l'obiettivo preposto era quello di valutare il ruolo del RA sintetizzato endogenamente su rigenerazione e differenziamento podocitario, senza, però, confonderlo con gli effetti immunomodulatori che tale molecola possiede (12). Parallelamente, il ruolo della somministrazione del RA è stato studiato in un ceppo murino transgenico, in cui si ha l'espressione del gene reporter LacZ, posto sotto il controllo di un RARE (Retinoic Acid Response Element), ossia una sequenza genomica a cui si lega il complesso formato da acido retinoico e relativi recettori intracellulari, al fine di attivare la trascrizione genica. Così facendo, è stato possibile visualizzare in blu quali cellule rispondessero al RA in vivo.

Gli studi condotti sul modello SCID hanno permesso agli Autori di dimostrare che il blocco della sintesi endogena di RA porta a un netto decremento nel numero di podociti e a un conseguente aumento di proteinuria e mortalità. Infatti, successivamente all'induzione di AN, gli animali trattati con un inibitore dell'enzima retinaldeide deidrogenasi ( $\mathrm{Di}$ sulfiram, DSF), necessario per la sintesi di acido retinoico, hanno mostrato una forte riduzione nella sopravvivenza, valori nettamente maggiori di albuminuria, maggiore glomerulosclerosi e un numero drasticamente inferiore di podociti rispetto al gruppo di controllo. Con ciò si dimostra come la sintesi endogena di acido retinoico limiti e prevenga l'albuminuria e la glomerulosclerosi, inducendo il differenziamento dei progenitori renali a podociti.

Come già dimostrato, il RA è, però, soggetto alla neutralizzazione ad opera dell'albumina come conseguenza del danno podocitario, evento che impedisce alle RPC di acquisire le caratteristiche proprie del podocita. Tuttavia, i progenitori renali, in vivo, possiedono tutte le componenti della pathway biosintetica del RA, e tale capacità sintetica risulta essere identica in topi sani o affetti da AN: le RPC possono quindi sintetizzare il RA endogenamente anche in vivo, in condizioni sia fisiologiche che patologiche. Se, però, sono valutati i livelli di retinolo e acido retinoico nelle urine di topi sani o affetti da nefropatia da adriamicina, emergono delle profonde differenze. Nello studio (11) si dimostra come, in relazione ai livelli di albuminuria, in topi sani una piccola quantità di retinolo, ma non di RA, sia rilevabile a livello urinario. Al contrario, in seguito all'induzione di AN e danno podocitario, i livelli di retinolo urinario aumentano propor- 
zionalmente al grado di severità dell'albuminuria, mentre il RA è rilevabile solo per valori di albuminuria inferiori a 300 $\mathrm{mg} / \mathrm{dL}$.

Con ciò è dimostrato che, conseguentemente a un insulto podocitario, la barriera di filtrazione glomerulare ormai alterata permette il transito del retinolo, plausibilmente con il fine biologico di permettere l'attivazione delle RPC a fini rigenerativi. $\mathrm{Al}$ contempo, però, i livelli urinari di acido retinoico non aumentano parallelamente a quelli del retinolo, ma, piuttosto, diminuiscono con il graduale incremento della proteinuria, confermando che il RA è progressivamente sequestrato dall'albumina.

Gli Autori hanno, quindi, pensato di contrastare l'effetto dannoso dell'albuminuria mediante somministrazione esogena di acido retinoico. Il RA è stato inoculato sottocute nel modello murino transgenico RARE-LacZ, così da poter valutare effetti e parametri pro-rigenerativi, e quali cellule ne siano responsabili rispondendo al RA.

Il trattamento con acido retinoico si è dimostrato capace di portare a una netta riduzione della proteinuria nel gruppo trattato rispetto al gruppo di controllo, così come il numero dei podociti è tornato ai valori basali valutati prima dell'induzione di AN. Interessante è anche riportare che, negli animali trattati con RA, erano presenti più cellule transizionali con fenotipo intermedio tra RPC e podocita, ed esprimenti marcatori di entrambe le popolazioni cellulari. Ciò è un'ulteriore conferma di come l'acido retinoico promuova il differenziamento dei progenitori renali verso il lineage podocitario.

Analogamente, in seguito al danno podocitario, la colorazione determinata dal gene reporter LacZ è osservabile in alcune RPC presenti sulla capsula di Bowman o che stanno entrando nel tuft glomerulare. La somministrazione di RA porta a un netto incremento dell'attività del gene LacZ nelle sole RPC, le quali, come conseguenza dello stimolo apportato dall'acido retinoico, acquistano marker tipici podocitari e differenziano. Al contrario, in topi affetti da AN trattati con solo veicolo, l'attività del gene LacZ è quasi totalmente assente o, se presente, va scomparendo all'incremento della proteinuria dell'animale. Questo prova come il RA, sequestrato dall'albumina, non sia più capace di legare i RARE presenti nei progenitori al fine di attivarli.

\section{Discussione}

Dal lavoro di Peired et al. (11) emerge chiaramente come la proteinuria e, in particolare, l'albuminuria siano non solo valori prognostici, ma anche, e soprattutto, fattori fortemente coinvolti nell'aggravare il danno glomerulare impedendo la rigenerazione podocitaria.

Con questo studio è finalmente possibile spiegare perché l'abbattimento della proteinuria possieda un effetto protettivo nei confronti della struttura glomerulare e del GFR (Glomerular Filtration Rate). L'impiego di terapie anti-proteinuriche permette l'azione dell'acido retinoico, il quale, non essendo sequestrato dall'albumina, può indurre il differenziamento delle RPC a podociti, processo alterato in modo dose-dipendente dalla stessa albumina. Analogamente, il blocco della sintesi di acido retinoico in modelli di FSGS porta a un calo nel numero dei podociti e a un aumento della proteinuria, evidenziando come la sintesi endogena di RA sia essenziale per il mantenimento del numero dei podociti e di una corretta architettura glomerulare.

L'osservazione forse di maggior importanza è che, in modelli murini di AN, il retinolo passa liberamente attraverso la barriera di filtrazione glomerulare ed è trasformato in RA grazie all'azione della retinaldeide deidrogenasi. Ciò porta all'attivazione dei progenitori renali inducendone il differenziamento a podociti. Ma, qualora il danno porti a un progressivo aumento della proteinuria, questo meccanismo rigenerativo viene meno a causa del sequestro attuato dall'albumina ai danni del RA. Ciò spiega anche perché, nonostante i podociti possano potenzialmente essere sostituiti dai progenitori renali presenti nella capsula di Bowman, la capacità rigenerativa che si osserva in pazienti affetti da CKD appaia notevolmente limitata (13): la loro proteinuria è il fattore che impedisce la corretta riorganizzazione della struttura glomerulare.

Malgrado ciò, il processo rigenerativo può essere ripristinato, in modelli murini, una volta che la capacità differenziativa a podociti delle RPC sia ristabilita mediante somministrazione di acido retinoico per via esogena, bypassando il ruolo neutralizzante che l'albumina esercita sull'acido retinoico. A sostegno dei dati presentati in questo studio (11), sono presenti in letteratura numerosi articoli in cui è riportato come la glomerulosclerosi possa regredire e il numero podocitario aumentare qualora la proteinuria sia ridotta mediante trattamento con ACE inibitori, sia in roditori $(14,15)$ che in uo$\operatorname{mini}(14,16,17)$.

Lo studio condotto da Peired et al. dimostra perché il trattamento con terapie anti-proteinuriche porti alla regressione delle lesioni tipiche della FSGS e a un aumento nel numero di podociti: la riduzione dell'albuminuria permette alle RPC di captare RA, attivarsi e dare luogo al rimodellamento che segue l'insulto iniziale e che permette la regressione del danno. Analogamente, se si neutralizza la barriera dell'albumina somministrando acido retinoico, i progenitori renali riescono nuovamente a differenziare per avviare il processo rigenerativo.

Di particolare rilievo, questo articolo fornisce il razionale biologico per l'impiego in ambito clinico di modulatori farmacologici che promuovano la regressione di glomerulopatie.

Tra tali modulatori, l'acido retinoico si ritaglia un nuovo proprio spazio e prospettive molto interessanti. Il RA potrebbe, infatti, stimolare le cellule staminali renali presenti fisiologicamente a dar luogo a eventi rigenerativi, strategia che sempre più sta prendendo piede nel campo della medicina rigenerativa. È, comunque, da considerare il rischio legato alla somministrazione di vitamina A e derivati, nonché agli eventuali effetti sistemici che tale terapia potrebbe comportare $(18,19)$. Per questo motivo, saranno necessari ulteriori studi affinché da tale scoperta di natura preclinica si possa passare all'attuazione clinica, valutando possibili strategie e conoscendo a fondo tutti gli eventi molecolari indotti dall'acido retinoico. 


\section{Riassunto}

Nella malattia renale cronica, il rischio di fallimento d'organo dipende dal grado di severità della proteinuria, la quale è determinata dal numero di podociti persi e dalla conseguente fibrosi glomerulare. Esistono, tuttavia, numerose evidenze cliniche e sperimentali che suggeriscono la possibilità di remissione della malattia renale e, in alcuni casi, persino di regressione del danno, quando ancora l'istologia dell'organo non risulti totalmente compromessa. Tali risultati sono ottenuti in particolare mediante l'impiego di terapie con effetti anti-proteinurici.

Nuove evidenze sperimentali suggeriscono perché il blocco della perdita di proteine urinarie permetta la remissione della malattia renale cronica. In un recente articolo di Peired et al., è, per la prima volta, dimostrato come l'albuminuria blocchi il processo rigenerativo a causa del sequestro attuato dall'albumina ai danni della vitamina A, noto agente differenziativo per popolazioni di progenitori staminali presenti in vari organi. La conseguente perdita della vitamina A, complessata all'albumina, con le urine, impedisce, quindi, l'attivazione dei progenitori renali residenti nella capsula di Bowman, bloccando sul nascere la risposta rigenerativa e permettendo la progressione della malattia renale cronica.

Parole chiave: Rigenerazione renale, Rigenerazione podocitaria, Albuminuria, Acido retinoico

Dichiarazione di conflitto di interessi: L'Autore dichiara di non avere conflitto di interesse.

Contributi economici agli Autori: L'Autore dichiara di non aver ricevuto sponsorizzazioni economiche per la preparazione dell'articolo.

\section{Indirizzo dell'Autore:}

Dr. Duccio Lombardi

Centro Denothe

Università degli Studi di Firenze

V.le G. Pieraccini 6

50139 Firenze

lombarduccio@alice.it

\section{Bibliografia}

1. Davies DJ, Brewer DB. Irreversible glomerular damage following heterologous serum albumin overload. J Pathol 1977; 123: 45-52.

2. Davies DJ, Messina A, Thumwood CM, Ryan GB. Glomerular podocyte injury in protein overload proteinuria. Pathology 1985; 17: 412-9.

3. Brewer DB, Filip O. The morphometry of the glomerular epithelial cell and its foot processes after the injection of bovine serum albumin or egg albumin. J Pathol 1976; 120: 209-20.

4. Davies DJ, Brewer DB, Hardwicke J. Urinary proteins and glomerular morphometry in protein overload proteinuria. Lab Invest 1978; 38: 232-43.

5. Ruggenenti P, Perna A, Mosconi L, et al. Proteinuria predicts end-stage renal failure in non-diabetic chronic nephropathies. The "Gruppo Italiano di Studi Epidemiologici in Nefrologia" (GISEN). Kidney Int Suppl 1997; 63: S54-7.

6. Remuzzi G. The nephropathic nature of proteinuria. Curr Opin Nephrol Hyperten 1999; 8: 655-3.

7. Sagrinati C, Netti GS, Mazzinghi B, et al. Isolation and characterization of multipotent progenitor cells from the Bowman's capsule of adult human kidneys. J Am Soc Nephrol 2006; 17: 2443-56.

8. Ronconi E, Sagrinati C, Angelotti ML, et al. Regeneration of glomerular podocytes by human renal progenitors. J Am Soc Nephrol 2009; 20: 322-32.

9. Remuzzi A, Ruggenenti P, Mosconi L, Pata V, Remuzzi G. Effect of low-dose enalapril on glomerular size selectivity in human diabetic nephropathy. J Nephrol 1993; 6: 36-43.

10. Brenner BM, Cooper ME, de Zeeuw D, et al. Effects of losartan on renal and cardiovascular outcomes in patients with type 2 diabetes and nephropathy. N Engl J Med 2001; 345: 861-9.

11. Peired A, Angelotti ML, Ronconi E, et al. Proteinuria impairs podocyte regeneration by sequestering retinoic acid. J Am Soc Nephrol 2013; 24 (11): 1756-68.

12. Shima Y, Iwano M, Yoshizaki K, Tanaka T, Kawase I, Nishimoto N. All-trans-RetinoicsonoAcid acid inhibits the development of mesangial proliferative glomerulonephritis in interleukin-6 transgenic mice. Nephron Exp Nephrol 2005; 100 (1): e54-62.

13. Lasagni L, Romagnani P. Glomerular epithelial stem cells: the good, the bad, and the ugly. J Am Soc Nephrol 2010; 21 (10): 1612-9.

14. Remuzzi G, Benigni A, Remuzzi A. Mechanisms of progression and regression of renal lesions of chronic nephropathies and diabetes. J Clin Invest 2006; 116 (2): 288-96.

15. Adamczak M, Gross ML, Krtil J, et al. Reversal of glomerulosclerosis after high-dose enalapril treatment in subtotally nephrectomized rats. J Am Soc Nephrol 2003; 14 (11): 2833-42.

16. Ruggenenti P, Perna A, Benini R, et al. In chronic nephropathies prolonged ACE inhibition can induce remission: dynamics of time-dependent changes in GFR. Investigators of the GISEN Group. Gruppo Italiano Studi Epidemiologici in Nefrologia. J Am Soc Nephrol 1999; 10 (5): 997-1006.

17. Wilmer WA, Hebert LA, Lewis EJ, et al. Remission of nephrotic syndrome in type 1 diabetes: long-term follow-up of patients in the Captopril Study. Am J Kidney Dis 1999; 34 (2): 308-14.

18. Bushue N, Wan YJ. Retinoid pathway and cancer therapeutics. Adv Drug Deliv Rev 2010; 62 (13): 1285-98.

19. Sporn MB, Roberts AB. Role of retinoids in differentiation and carcinogenesis. Cancer Res 1983; 43 (7): 3034-40. 